POS PROCEEDINGS

\title{
Next generation jet measurements with sPHENIX
}

\section{Songkyo Lee for the sPHENIX collaboration*}

Iowa State University, Ames, IA 50010, USA

E-mail: songkyo.leedcern.sh

\begin{abstract}
The proposed sPHENIX detector at the Relativistic Heavy Ion Collider (RHIC), designed to take advantage of technological advances from the Large Hadron Collider (LHC), will allow measurements of jets and jet correlations with a kinematic reach that will overlap with measurements made at the LHC. This is made possible by taking advantage of the increased luminosity due to accelerator upgrades and the ability of sPHENIX to sample the entire luminosity. Particle jets, formed when a hard scattered parton fragments and then hadronizes into a spray of particles, are an excellent as a probe of the Quark Gluon Plasma (QGP) formed in heavy-ion collisions. As these partons traverse the QGP formed in heavy ion collisions, they lose energy to the medium, in a process called jet quenching. The physics of this process can be explored by comparing jetbased observables in heavy-ion collisions to those in proton-proton collisions. This is necessary in order to answer the fundamental questions of how and why partons lose energy in the QGP, which will require that we characterize both the medium induced modification of the jet fragmentation pattern, and the correlation of the lost energy with the jet axis. Observables which use the correlation between a high energy photon and a jet are especially useful as the photon kinematics are more closely correlated with the parton kinematics, as well as preferentially selecting quark jets over gluon jets. In addition to inclusive or semi-inclusive jet observables, advances in both theory and measurement at the LHC have provided a suite of new observables which are related to the fragmentation pattern of the jet and how it is modified in the QGP medium. These observables require the precise tracking and calorimetry that the sPHENIX design has. We will show the performance of jet and gamma-jet observables within the sPHENIX detector, including the jet energy scale and resolution in both proton-proton and heavy-ion collisions. These results are simulated with the improved framework developed for understanding the performance of the new detector for measuring jets and photons in a heavy ion environment.
\end{abstract}

International Conference on Hard and Electromagnetic Probes of High-Energy Nuclear Collisions 30 September - 5 October 2018

Aix-Les-Bains, Savoie, France

${ }^{*}$ Speaker. 


\section{Introduction}

Back in 2015 in the Nuclear Science Long Range Plan [1], sPHENIX is newly proposed at the Relativistic Heavy Ion Collider (RHIC) to probe the inner workings of Quark-Gluon Plasma (QGP) created in ultra-relativistic heavy ion collisions, at a wide range of length scales. It will be a stateof-the-art jet detector that can provide fully calorimetric jet measurements at RHIC. The superior acceptance and collider performance will also enable us to access wider kinematic ranges and have precise measurements complementary to those at the Large Hadron Collider (LHC), mapping out the QGP in different temperature and density.

The sPHENIX collaboration was formed in 2015 and granted CD-0 next year, the first stage of official project approval from the US Department of Energy (DOE). In 2017, sPHENIX got CD$1 / 3 \mathrm{~A}$ that approves cost range, advanced purchase, and project schedule. Detector installation and commisioning are expected to be complete in 2022, and the first physics data will be taken in 2023. The multiyear run plan includes collisions of various species (p+p, p+A, and $\mathrm{Au}+\mathrm{Au}$ ) at $200 \mathrm{GeV}$. Approximately 239 billion events of $\mathrm{Au}+\mathrm{Au}$ minimum bias data will be recorded in total.

The key physics programs planned at sPHENIX are jet substructure, photon-jet correlations, upsilon spectroscopy, and heavy-flavor measurements. In this presentation, simulation of jet physics performance is reported.

\section{Detector Design}

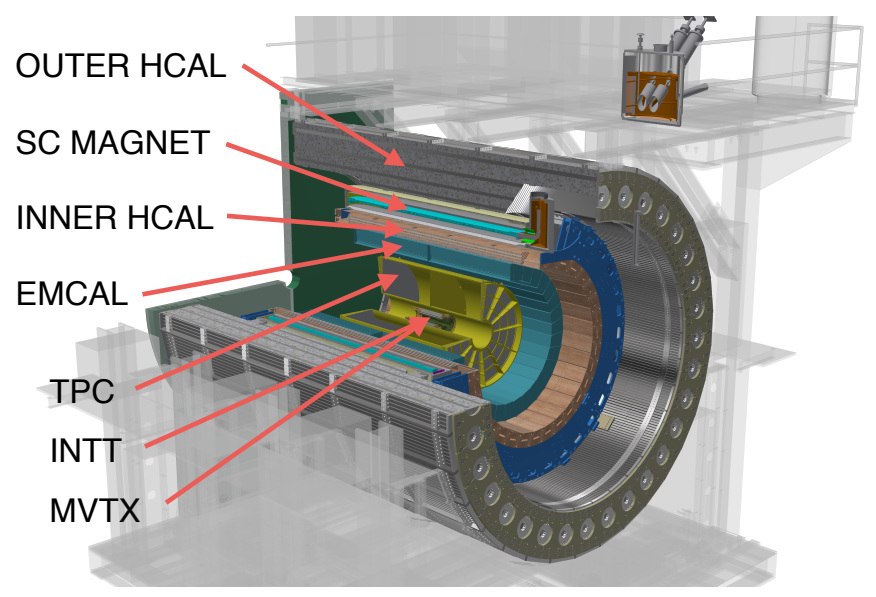

Figure 1: A schematic view of the sPHENIX detector with its subdetectors labeled.

The sPHENIX detector, shown in Fig. 1, has the full azimuthal acceptance of $0<\phi<2 \pi$ and uniformly covers the pseudorapidity range of $|\eta|<0.85$. The innermost part of the sPHENIX is the tracking system composed of Monolithic Active Pixel Vertex Detector (MVTX), Silicon Strip Intermediate Tracker (INTT), and Time Projection Chamber (TPC). The electromagnetic calorimeter (EMCal) consists of Tungsten towers with embedded scintillating fibers and has a high degree of segmentation $(\eta \times \phi=0.025 \times 0.025)$. The inner (Inner HCal) and outer hadronic calorimeters (Outer $\mathrm{HCal}$ ) are located inside and outside the $1.5 \mathrm{~T}$ superconducting magnetic solenoid, respectively. Currently, various options are being considered for Inner HCal, e.g., Aluminum Frame with 
or without being read out. The Outer $\mathrm{HCal}$ is a sampling calorimeter with alternating layers of steel plates and scintillating tiles. It also serves as the magnet flux return. The granularity of $\mathrm{HCal}$ towers is $\eta \times \phi=0.1 \times 0.1$. More details can be found in Ref. [2].

In order to perform desired jet measurements, the calorimeter system must satisfy a set of physics-driven requirements. Jet energy resolution needs to be $\sigma_{E} / E<120 \% / \sqrt{E}$ in p+p and $\sigma_{E} / E<150 \% / \sqrt{E}$ in Au+Au collisions. For photon-jet correlation measurements, photon energy resolution in EMCal is required to be $\sigma_{E} / E<15 \% / \sqrt{E}$. For fragmentation function measurements, excellent tracking resolution is needed, e.g., $d p / p<0.2 \% \times p$ for a $40 \mathrm{GeV}$ jet.

\section{Simulation of Jet Performance}

To establish the capabilities of the sPHENIX calorimetry system for jet measurements, a full GEANT4 set of simulations [3] has been performed. Jets are generated using PYTHIA8 [4] to simulate the $\mathrm{p}+\mathrm{p}$ collisions at $200 \mathrm{GeV}$ and then reconstructed by clustering calorimeter towers with the anti- $k_{\mathrm{T}}$ jet finding algorithm in the FastJet package [5], with the resolution parameter $R=0.2$ and 0.4 . To simulate $\mathrm{Au}+\mathrm{Au}$ collisions, PYTHIA8 signals are embedded into HIJING simulated background events [6]. Truth jets are determined by putting generated particles through the same FastJet package, and selected to be in the central region of $|\eta|<0.45$.

One of the most important steps in heavy-ion jet reconstruction is the underlying event (UE) subtraction. An iterative UE subtraction algorithm has been used based on the PHENIX and ATLAS method [7]. First, jets are reconstructed using raw calorimeter towers, and the seed jets are selected if $\operatorname{Max}\left(E_{\mathrm{T}}^{\text {tower }}\right) / \operatorname{Mean}\left(E_{\mathrm{T}}^{\text {tower }}\right)>D$, where $D$ is the threshold value. The background is defined in each calorimeter layer and $\eta$-ring, after excluding the seed jet region. Next, the $\Psi_{2}$ and $v_{2}$ are determined from $\eta$-rings with full $\phi$ coverage. Finally, backgrounds are subtracted towerby-tower modulating the determined flow. For the second iteration, the seed jet is determined by $E_{\mathrm{T}}$ threshold. The jet finder is run over the background-subtracted events to find final jets.
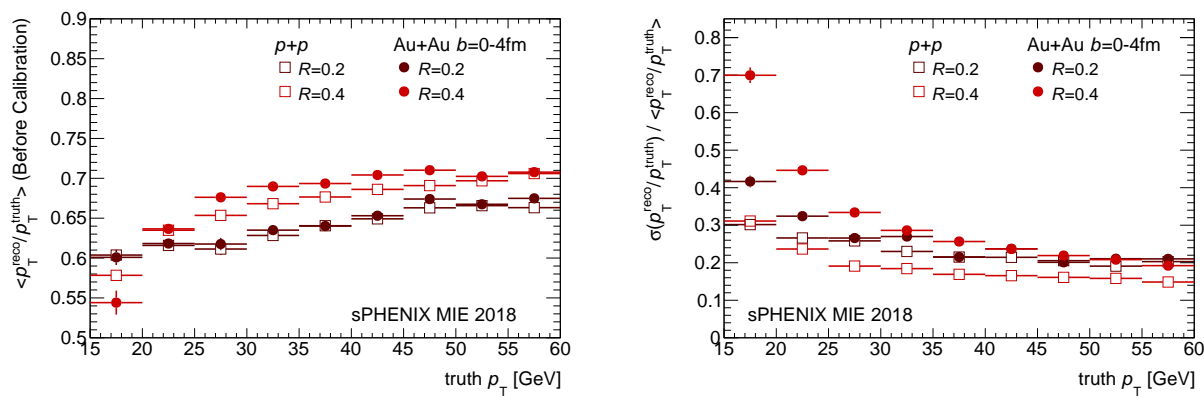

Figure 2: Jet Energy Scale (left) and Jet Energy Resolution (right) as a function of truth jet $p_{\mathrm{T}}$ for $R=0.2$ and $R=0.4$ jets in simulated $\mathrm{p}+\mathrm{p}$ and central $\mathrm{Au}+\mathrm{Au}$ events. (Inner $\mathrm{HCal}$ is not instrumented in this simulation.)

Figure 2 shows the Jet Energy Scale (JES) on the left, and Jet Energy Resolution (JER) on the right as a function of truth jet $p_{\mathrm{T}}$. Inner $\mathrm{HCal}$ is not instrumented in this simulation. The JES follows the expected ordering in $R$, and similar in $\mathrm{p}+\mathrm{p}$ and $\mathrm{Au}+\mathrm{Au}$ events independent of UE level. The JER is dominated by fluctuations in UE subtraction at large $R$ and low $p_{\mathrm{T}}$ and therefore larger for larger $R$ in $\mathrm{Au}+\mathrm{Au}$ collisions. At small $R$ or high $p_{\mathrm{T}}$, JER is dominated by an intrinsic resolution of calorimeters, so the JER values in $\mathrm{p}+\mathrm{p}$ and $\mathrm{Au}+\mathrm{Au}$ collisions are rather comparable. 
It is worth noting that the EMCal is calibrated for pure electromagnetic (EM) energy in Fig. 2, but the EMCal has a different response to EM and hadronic showers. Also, the response of calorimeters to a jet depends on the longitudinal center of gravity, the position at which a shower begins to develop. It indicates that the EM and hadronic energy deposit in different calorimeter segments needs to be calibrated separately. The reconstructed jet energy can be expressed as:

$$
E_{\mathrm{Jet}}^{\mathrm{Reco}}=D(E) \cdot\left[E_{\mathrm{EMCal}}^{\mathrm{em}}+A(E) \cdot E_{\mathrm{EMCal}}^{\mathrm{had}}+B(E) \cdot E_{\text {InnerHCal }}+C(E) \cdot E_{\text {OuterHCal }}\right],
$$

where $E_{\mathrm{EMCal}}^{\mathrm{em}}$ and $E_{\mathrm{EMCal}}^{\mathrm{had}}$ are the deposited energy in the EMCal classified as electromagnetic and hadronic, respectively. Similarly, $E_{\text {InnerHCal }}$ and $E_{\text {OuterHCal }}$ are the deposited energy in the Inner $\mathrm{HCal}$ and the Outer $\mathrm{HCal}$. The coefficients $A, B, C$, and $D$ are calibration factors and determined by minimizing the quantity, $\Sigma_{i=1}^{N}\left(E_{\mathrm{Jet}, i}^{\mathrm{Reco}}-E_{\mathrm{Jet}, i}^{\text {Truth }}\right) / E_{\mathrm{Jet}, i}^{\text {Truth }}$, using the numerical minimization computer program, MINUIT2 [8].

Figure 3 shows the JER as a function of truth jet $p_{\mathrm{T}}$ before and after the calibration scheme is applied, for $R=0.2$ jets on the left and $R=0.4$ jets on the right. Shaded boxes represent uncertainties in the calibration factors. Inner HCal is instrumented in this simulation. There is an improvement in JER after calibration over the whole $p_{\mathrm{T}}$ range, both in $\mathrm{p}+\mathrm{p}$ and $\mathrm{Au}+\mathrm{Au}$ events.
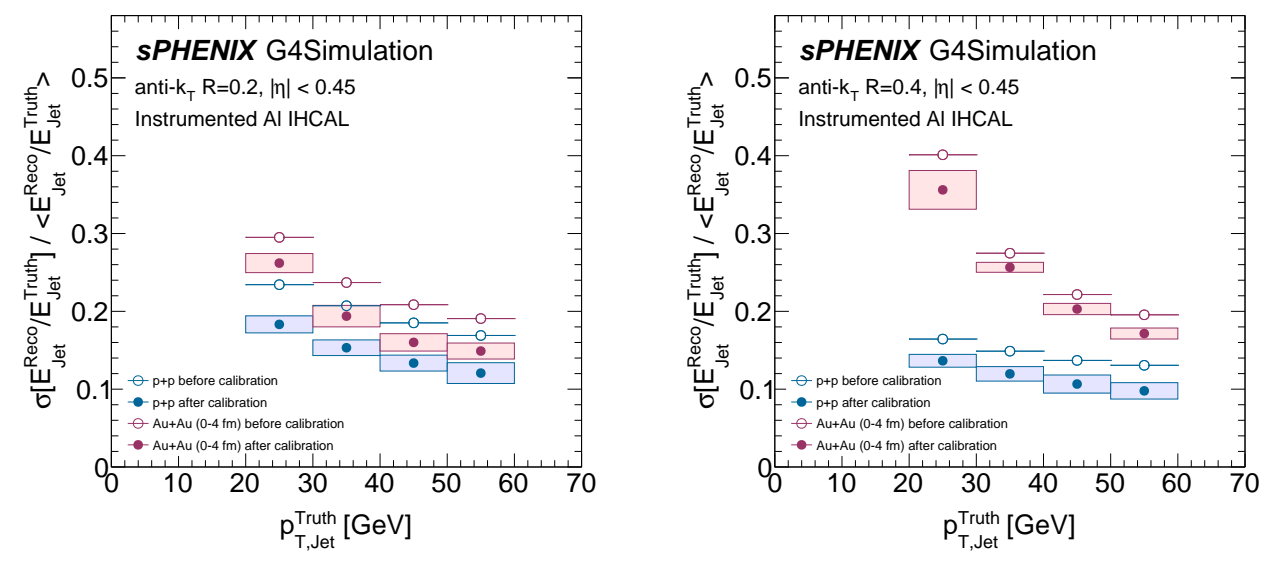

Figure 3: Jet Energy Resolution as a function of truth jet $p_{\mathrm{T}}$ for $R=0.2$ (left) and $R=0.4$ (right) jets in simulated $\mathrm{p}+\mathrm{p}$ and central $\mathrm{Au}+\mathrm{Au}$ events. Open and filled markers are the results before and after the calorimeter-segment calibration, respectively. (Inner $\mathrm{HCal}$ is instrumented in this simulation.)

As well as the jet reconstruction, the photon isolation algorithm also has been developed, which is important for photon-jet correlation measurements. First, an EMCal cluster above certain $E_{\mathrm{T}}$ value is selected as a photon candidate. Then, $E_{\mathrm{T}}$ of all the towers around this cluster is summed over within the isolation cone $\Delta R$. The reconstructed isolation energy is defined by $E_{\mathrm{T}}^{\text {iso }}=\left(\Sigma E_{\mathrm{T}}^{\text {Tower }}\right)-E_{\mathrm{T}}^{\text {Cluster }}$. Currently, sPHENIX clustering and cluster-splitting algorithms utilize the shower profile based on the PHENIX method.

Figure 4 shows the $E_{\mathrm{T}}^{\text {iso }}$ distributions for different $\Delta R$ in central $\mathrm{Au}+\mathrm{Au}$ events. Inner $\mathrm{HCal}$ is not instrumented in this simulation. Filled points are the results with raw towers, and $E_{\mathrm{T}}^{\text {iso }}$ is centered around higher values for larger $\Delta R$ due to the presence of UE. As a next step, the photon isolation is performed over the UE-subtracted towers, as it is done for jets. The UE-subtracted results are shown as open points, and $E_{\mathrm{T}}^{\text {iso }}$ is peaking at zero for all $\Delta R$. Distributions are broader 
for larger $\Delta R$ as the background fluctuations are expected to be proportional to the isolation cone size. By this study, it can also be validated that the UE subtraction is working reasonably.

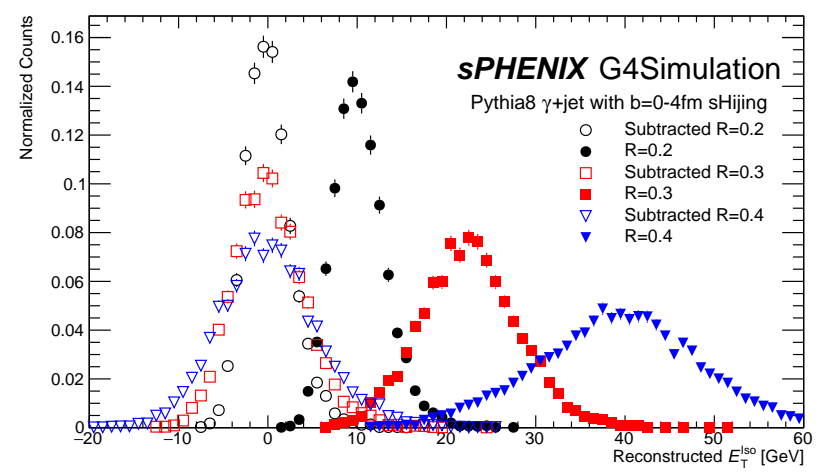

Figure 4: Distributions of $\mathrm{E}_{\mathrm{T}}^{\text {iso }}$ for different isolation cone size $\Delta R$ in simulated $\mathrm{Au}+\mathrm{Au}$ events. Filled points are the results with raw towers, and open points are with the UE-subtracted towers. (Inner HCal is not instrumented in this simulation.)

\section{Summary}

The sPHENIX detector will allow measurements of fully calorimetric jets for the first time at RHIC, with an extended kinematic reach complementary to that of measurements at the LHC. The sPHENIX project is essential to understanding the underlying dynamics of QGP in heavy-ion collisions, including the microscopic behavior.

The simulation has been done to study the performance of jet and photon measurements with realistic sPHENIX detector framework. The JES and JER, the jet energy calibration scheme, and the photon isolation in $\mathrm{p}+\mathrm{p}$ and central $\mathrm{Au}+\mathrm{Au}$ events have been presented. The simulated results indicate that the current sPHENIX design meets our physics requirements for jets.

\section{References}

[1] A Aprahamian et al. Reaching for the horizon: The 2015 long range plan for nuclear science, 2015

[2] A. Adare et al. An Upgrade Proposal from the PHENIX Collaboration, 2015

[3] S. Agostinelli et al. GEANT4: A Simulation toolkit, Nucl.Instrum.Meth. A 506 (2003) 250

[4] T. Sjostrand, S. Mrenna, P. Skands, A Brief Introduction to PYTHIA 8.1, Comput.Phys.Commun. 178 (2008) 852

[5] M. Cacciari, G. P. Salam, G. Soyez, FastJet user manual, Eur.Phys.J. C 72 (2012) 1896

[6] X. Wang, M. Gyulassy, HIJING 1.0: A Monte Carlo Program for Parton and Particle Production in High Energy Hadronic and Nuclear Collisions, Comput.Phys. Commun. 83 (1994) 307

[7] J.A. Hanks et al. Method for separating jets and the underlying event in heavy ion collisions at the BNL Relativistic Heavy Ion Collider, Phys.Rev. C 86 (2012) 024908

[8] F. James, M. Roos, Minuit: A System for Function Minimization and Analysis of the Parameter Errors and Correlations, Comput.Phys.Commun. 10 (1975) 343 